\title{
Variant $\mathcal{N}=(1,1)$ supergravity and $(\text { Minkowski })_{4} \times S^{2}$ vacua
}

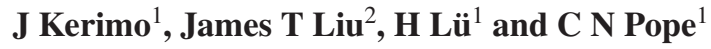 \\ ${ }^{1}$ George P and Cynthia W Mitchell Institute for Fundamental Physics, Texas A \& M University, \\ College Station, TX 77843-4242, USA \\ ${ }^{2}$ Michigan Center for Theoretical Physics, University of Michigan, Ann Arbor, MI 48109-1120, \\ USA
}

Received 25 February 2004

Published 10 June 2004

Online at stacks.iop.org/CQG/21/3287

doi:10.1088/0264-9381/21/13/011

\begin{abstract}
We construct the fermionic sector and supersymmetry transformation rules of a variant $\mathcal{N}=(1,1)$ supergravity theory obtained by generalized KaluzaKlein reduction from seven dimensions. We show that this model admits both (Minkowski) ${ }_{4} \times S^{2}$ and (Minkowski) $)_{3} \times S^{3}$ supersymmetric vacua. We perform a consistent Kaluza-Klein reduction on $S^{2}$ and obtain $D=4, \mathcal{N}=2$ supergravity coupled to a vector multiplet, which can be consistently truncated to give rise to $D=4, \mathcal{N}=1$ supergravity with a chiral multiplet.
\end{abstract}

PACS numbers: 04.65.+e, 11.30.Pb, 11.25.Mj

\section{Introduction}

The notion of higher dimensional theories and their Kaluza-Klein compactifications have played a central role in many modern approaches to unification, and especially M-theory. In such models, it is essential on phenomenological grounds that the resulting lower dimensional theory yields a realistic spectrum of chiral fermions as well as correct strength couplings and a small (or vanishing) cosmological constant. Thus while spheres appear to be natural and simple candidates for an internal space, they are often rejected as they would typically give rise to a lower dimensional theory with a large cosmological constant (directly related to the curvature of the sphere).

However, a rare example of a sphere reduction admitting a flat Minkowski spacetime has been known for a while. This is the Salam-Sezgin model [1], which is essentially gauged $\mathcal{N}=(1,0)$ supergravity in six dimensions admitting a (Minkowski) $)_{4} \times S^{2}$ vacuum. This model has the added feature that the vanishing of the cosmological constant in four dimensions typically arises naturally on the basis of an interplay between the six-dimensional potential and the $U(1)$ monopole flux on the $S^{2}$. This self-tuning mechanism has been 
highlighted recently in [2, 3] where it was noted that the four-dimensional cosmological constant is protected against large contributions in a Salam-Sezgin braneworld scenario, even after supersymmetry breaking on the branes. (Note, however, that this self-tuning presupposes the existence of a (Minkowski) 4 vacuum to start with [4].) The $S^{2}$ reduction of the Salam-Sezgin model was recently examined in [5], and its lower dimensional spectrum was analysed.

Recently a variant $\mathcal{N}=(1,1)$ supergravity in $D=6$ was obtained from generalized Kaluza-Klein reduction; as in the Salam-Sezgin model, this theory also admits a (Minkowski) $)_{4} \times S^{2}$ vacuum solution [6]. Unlike the Salam-Sezgin model, however, the variant $\mathcal{N}=(1,1)$ supergravity includes vector fields in the gravity multiplet itself. In this case, the turning on of a six-dimensional potential is related to mass generation for one of the graviphotons. The bosonic equations of motion for this model were obtained in [6], where it was further argued that the model is indeed supersymmetric. In this paper, we complete the reduction of [6] and prove its supersymmetry by obtaining a complete set of supersymmetry transformation rules via Kaluza-Klein reduction of the original $D=7$ theory.

After obtaining the complete supersymmetry transformations of the variant $\mathcal{N}=(1,1)$ theory, we investigate some of its spontaneous compactifications. As in the $\mathcal{N}=(1,0)$ model, we find that it can also be consistently reduced on a 2-sphere to give rise to four-dimensional $\mathcal{N}=2$ supergravity coupled to a single vector multiplet. This can be further truncated to yield $\mathcal{N}=1$ supergravity coupled to a chiral multiplet. We further demonstrate that, in contrast to the $\mathcal{N}=(1,0)$ theory, this model also admits a supersymmetric (Minkowski) ${ }_{3} \times S^{3}$ vacuum.

We begin in section 2 by investigating the fermionic sector of the $D=7$ to $D=6$ reduction. After constructing the fermionic reduction ansatz, we provide the complete supersymmetry transformations of the variant $\mathcal{N}=(1,1)$ supergravity. In section 3 , we examine the reduction to (Minkowski) $)_{4} \times S^{2}$. Since the resulting theory is simply $\mathcal{N}=2$ supergravity coupled to a vector, it naturally admits supersymmetric dyonic black-hole solutions. The black hole and its elevation back to six dimensions is given in section 4 . Finally, the (Minkowski) $)_{3} \times S^{3}$ vacuum is investigated in section 5 , and we end with a discussion on four-dimensional chirality in section 6 .

\section{Supersymmetry of the generalized reduction}

The bosonic field content of half-maximum supergravity in seven dimensions comprises a metric $\hat{g}_{M N}$, a scalar $\hat{\phi}$, an antisymmetric tensor $\hat{B}_{(2)}$ and three vectors $\hat{A}_{(1)}^{a}$. The Lagrangian in the bosonic sector is $[7,8]$

$\hat{\mathcal{L}}=\hat{R} \hat{*} \mathbb{1}-\frac{1}{2} \hat{*} \mathrm{~d} \hat{\phi} \wedge \mathrm{d} \hat{\phi}-\frac{1}{2} \mathrm{e}^{\frac{4}{\sqrt{10}} \hat{\phi}} \hat{*} \hat{H}_{(3)} \wedge \hat{H}_{(3)}-\frac{1}{2} \mathrm{e}^{\frac{2}{\sqrt{10}} \hat{\phi}} \hat{*} \hat{F}_{(2)}^{a} \wedge \hat{F}_{(2)}^{a}$,

where $\hat{F}_{(2)}^{a}=\mathrm{d} \hat{A}_{(1)}^{a}$ and $\hat{H}_{(3)}=\mathrm{d} \hat{B}_{(2)}-\frac{1}{2} \hat{F}_{(2)}^{a} \wedge \hat{A}_{(1)}^{a}$.

\subsection{The supersymmetry transformations}

The fermionic sector consists of a pair of symplectic-Majorana gravitinos $\hat{\psi}_{M i}$ as well as a pair of dilatinos $\hat{\lambda}_{i}$, where $i=1,2$ is an $\operatorname{Sp}(1)$ index. The three vectors form a triplet under $\operatorname{Sp}(1)$, and may equivalently be written as $\hat{A}_{(1) i}{ }^{j}=\hat{A}_{(1)}^{a}\left(-\tau^{a}\right)_{i}{ }^{j}$ where $\tau^{a}$ are the usual Pauli matrices. In this form, the supersymmetry transformations on the fermions are given by 


$$
\begin{aligned}
\delta \hat{\psi}_{M i}=\left[\hat{\nabla}_{M}\right. & \left.-\frac{1}{60}\left(\hat{\gamma}_{M}^{N P Q}-\frac{9}{2} \delta_{M}^{N} \hat{\gamma}^{P Q}\right) \mathrm{e}^{\frac{1}{2} \hat{a} \hat{\phi}} \hat{H}_{N P Q}\right] \hat{\epsilon}_{i} \\
& +\frac{\mathrm{i}}{20 \sqrt{2}}\left(\hat{\gamma}_{M}^{N P}-8 \delta_{M}^{N} \hat{\gamma}^{P}\right) \mathrm{e}^{\frac{1}{4} \hat{a} \hat{\phi}} \hat{F}_{N P i}{ }^{j} \hat{\epsilon}_{j}, \\
\delta \hat{\lambda}_{i}= & {\left[-\frac{1}{2 \sqrt{2}} \hat{\gamma}^{M} \partial_{M} \hat{\phi}+\frac{1}{12 \sqrt{5}} \mathrm{e}^{\frac{1}{2} \hat{a} \hat{\phi}} \hat{H}_{M N P} \hat{\gamma}^{M N P}\right] \hat{\epsilon}_{i}-\frac{\mathrm{i}}{4 \sqrt{10}} \mathrm{e}^{\frac{1}{4} \hat{a} \hat{\phi}} \hat{F}_{M N i}{ }^{j} \hat{\gamma}^{M N} \hat{\epsilon}_{j}, }
\end{aligned}
$$

where $\hat{a}=4 / \sqrt{10}$.

In addition, the transformations on the bosonic fields have the form

$$
\begin{aligned}
& \delta \hat{\phi}=-\frac{1}{2 \sqrt{2}} \overline{\hat{\epsilon}}^{i} \hat{\lambda}_{i}, \\
& \delta \hat{g}_{M N}=\frac{1}{2} \hat{\epsilon}^{i} \hat{\gamma}_{(M} \hat{\psi}_{N) i}, \\
& \delta \hat{A}_{M i}^{j}=\frac{\mathrm{i}}{\sqrt{2}} \mathrm{e}^{-\frac{1}{4} \hat{a} \hat{\phi}}\left(\overline{\hat{\psi}}^{j}{ }_{M}-\frac{1}{\sqrt{5}} \overline{\hat{\lambda}}^{j} \hat{\gamma}_{M}\right) \hat{\epsilon}_{i}, \\
& \delta \hat{B}_{M N}=-\frac{1}{2} \hat{A}_{[M i}{ }^{j} \delta \hat{A}_{N] j}{ }^{i}-\frac{1}{2} \mathrm{e}^{-\frac{1}{2} \hat{a} \hat{\phi}}\left(\overline{\hat{\psi}}^{i}{ }_{[M} \hat{\gamma}_{N]}-\frac{1}{\sqrt{5}} \overline{\hat{\lambda}}^{i} \hat{\gamma}_{M N}\right) \hat{\epsilon}_{i},
\end{aligned}
$$

where in the transformation for $\hat{A}_{M i}{ }^{j}$, the $\operatorname{Sp}(1)$ indices $i$ and $j$ are to be taken in the triplet combination. In particular, this may be enforced by the projection $\left(\delta_{i}^{i^{\prime}} \delta_{j^{\prime}}^{j}-\frac{1}{2} \delta_{i}^{j} \delta_{j^{\prime}}^{i^{\prime}}\right)$ which removes the trace. Note that the transformation for $\delta \hat{B}_{M N}$ is given in a dualized form compared to that of [7].

The above fermionic (2.2) and bosonic (2.3) supersymmetries are normalized according to

$\left[\delta_{1}, \delta_{2}\right] \hat{\boldsymbol{\Xi}}=\frac{1}{4} \hat{\xi}^{M} \partial_{M} \hat{\boldsymbol{\Xi}}+($ local Lorentz $)+($ general coordinate $)+($ gauge $)$,

where $\hat{\xi}^{M}=\overline{\hat{\epsilon}}_{2}^{i} \hat{\gamma}^{M} \hat{\epsilon}_{1 i}$. Furthermore, when working with the fermions, it is often convenient to make use of the Majorana flip conditions

$$
\begin{aligned}
\overline{\hat{\chi}}^{i} \gamma_{M_{1} M_{2} \cdots M_{n}} \hat{\psi}_{i} & =(-)^{n} \overline{\hat{\psi}}^{i} \gamma_{M_{n} M_{n-1} \cdots M_{1}} \hat{\chi}_{i}, \\
\overline{\hat{\chi}}^{j} \gamma_{M_{1} M_{2} \cdots M_{n}} \hat{\psi}_{i} & =(-)^{n+1} \overline{\hat{\psi}}^{j} \gamma_{M_{n} M_{n-1} \cdots M_{1}} \hat{\chi}_{i},
\end{aligned}
$$

for the singlet and triplet combinations, respectively.

\subsection{The bosonic reduction ansatz}

As demonstrated in [6], the generalized $S^{1}$ reduction ansatz is given on the bosonic fields by

$$
\begin{aligned}
& \mathrm{d} \hat{s}_{7}^{2}=\mathrm{e}^{2 m_{2} z}\left(\mathrm{e}^{2 \alpha \varphi} \mathrm{d} s_{6}^{2}+\mathrm{e}^{2 \beta \varphi}\left(\mathrm{d} z+\mathcal{A}_{(1)}\right)^{2}\right), \\
& \hat{B}_{(2)}=\mathrm{e}^{2\left(m_{2}-m_{1}\right) z}\left(B_{(2)}+B_{(1)} \wedge \mathrm{d} z\right), \\
& \hat{A}_{(1)}^{a}=\mathrm{e}^{\left(m_{2}-m_{1}\right) z}\left(A_{(1)}^{a}+\Phi^{a} \mathrm{~d} z\right), \\
& \hat{\phi}=\phi+\sqrt{10} m_{1} z,
\end{aligned}
$$

where $\alpha^{2}=\frac{1}{40}$ and $\beta=-4 \alpha$. The resulting reduction yields the six-dimensional fields $\left(g_{\mu \nu}, \mathcal{A}_{(1)}, A_{(1)}^{a}, B_{(2)}, \phi_{1}\right)$ and $\left(B_{(1)}, \phi_{2}, \Phi^{a}\right)$ corresponding to the bosonic content of $\mathcal{N}=$ $(1,1)$ supergravity coupled to a vector multiplet. Note that the $\operatorname{Sp}(1)$ singlet graviphoton and the matter vector are in actuality given by linear combinations of $\mathcal{A}_{(1)}$ and $B_{(1)}$. However, the scalars $\phi_{1}$ and $\phi_{2}$, given by the rotated combinations

are diagonal between multiplets.

$$
\phi_{1}=\frac{2}{\sqrt{5}} \phi-\frac{1}{\sqrt{5}} \varphi, \quad \phi_{2}=\frac{1}{\sqrt{5}} \phi+\frac{2}{\sqrt{5}} \varphi,
$$




\subsection{The fermionic reduction}

Working out the fermion reduction is straightforward, although somewhat tedious. Since the resulting $D=6$ theory contains a vector multiplet in addition to the pure supergravity multiplet, the $D=7$ fermions $\hat{\psi}_{M i}$ and $\hat{\lambda}_{i}$ must reduce to yield a $D=6$ gravitino and dilatino $\left(\psi_{\mu i}, \lambda_{i}\right)$ as well as a gaugino $\chi_{i}$. The reduction from seven to six dimensions is facilitated by the fact that the $D=7$ symplectic-Majorana condition $\overline{\hat{\psi}}^{i}=-\epsilon^{i j} \hat{\psi}_{j}^{T} \hat{C}$ continues to apply in $D=6$, yielding a trivial reduction on the spinors.

Examination of the supersymmetry transformations on the fermions, (2.2), indicates that the proper fermionic reduction is given by

$$
\begin{aligned}
& \hat{\epsilon}_{i}=\mathrm{e}^{\frac{1}{2} m_{2} z} \mathrm{e}^{\frac{1}{2} \alpha \varphi} \epsilon_{i}, \\
& \hat{\lambda}_{i}=\frac{1}{\sqrt{5}} \mathrm{e}^{-\frac{1}{2} m_{2} z} \mathrm{e}^{-\frac{1}{2} \alpha \varphi}\left(\chi_{i}+2 \lambda_{i}\right), \\
& \hat{\psi}_{z i}=\frac{2}{5} \mathrm{e}^{\frac{1}{2} m_{2} z} \mathrm{e}^{\left(\beta-\frac{1}{2} \alpha\right) \varphi} \gamma_{7}\left(2 \chi_{i}-\lambda_{i}\right), \\
& \hat{\psi}_{\mu i}=\mathrm{e}^{\frac{1}{2} m_{2} z} \mathrm{e}^{\frac{1}{2} \alpha \varphi}\left[\psi_{\mu i}+\left(\frac{2}{5} \mathrm{e}^{(\beta-\alpha) \varphi} \mathcal{A}_{\mu} \gamma_{7}-\frac{1}{10} \gamma_{\mu}\right)\left(2 \chi_{i}-\lambda_{i}\right)\right] .
\end{aligned}
$$

In this case, the resulting $D=6$ fermions have supersymmetry transformations

$$
\begin{aligned}
& \delta \psi_{\mu i}=\left[\nabla_{\mu}-\frac{5}{8} m_{2} \gamma_{\mu} \gamma^{\nu} \mathcal{A}_{\nu}-\frac{1}{48} \mathrm{e}^{\frac{1}{\sqrt{2}} \phi_{1}}\left(\gamma_{\mu}^{\nu \rho \sigma}-3 \delta_{\mu}^{\nu} \gamma^{\rho \sigma}\right) H_{\nu \rho \sigma}+\frac{5}{8} m_{2} \mathrm{e}^{\frac{1}{\sqrt{2}} \phi_{2}-\frac{1}{2 \sqrt{2}} \phi_{1}} \gamma_{\mu} \gamma_{7}\right. \\
& \left.-\frac{1}{32} \mathrm{e}^{\frac{1}{2 \sqrt{2}} \phi_{1}}\left(\gamma_{\mu}^{\nu \rho}-6 \delta_{\mu}^{\nu} \gamma^{\rho}\right) \gamma_{7}\left(\mathrm{e}^{\frac{1}{\sqrt{2}} \phi_{2}} H_{\nu \rho}+\mathrm{e}^{-\frac{1}{\sqrt{2}} \phi_{2}} \mathcal{F}_{\nu \rho}\right)\right] \epsilon_{i} \\
& +\left[-\frac{\mathrm{i}}{2 \sqrt{2}} \mathrm{e}^{\frac{1}{\sqrt{2}} \phi_{2}} \gamma_{7} Q_{\mu i}{ }^{j}+\frac{\mathrm{i}}{16 \sqrt{2}} \mathrm{e}^{\frac{1}{2 \sqrt{2}} \phi_{1}}\left(\gamma_{\mu}{ }^{\nu \rho}-6 \delta_{\mu}^{\nu} \gamma^{\rho}\right) F_{\nu \rho i}{ }^{j}\right] \epsilon_{j}, \\
& \delta \lambda_{i}=\left[-\frac{1}{2 \sqrt{2}} \gamma^{\mu} \partial_{\mu} \phi_{1}+\frac{1}{4}\left(4 m_{1}+m_{2}\right) \mathcal{A}_{\mu} \gamma^{\mu}+\frac{1}{24} \mathrm{e}^{\frac{1}{\sqrt{2}} \phi_{1}} H_{\mu \nu \rho} \gamma^{\mu \nu \rho}\right. \\
& +\frac{1}{16} \mathrm{e}^{\frac{1}{2 \sqrt{2}} \phi_{1}} \gamma^{\mu \nu} \gamma_{7}\left(\mathrm{e}^{\frac{1}{\sqrt{2}} \phi_{2}} H_{\mu \nu}+\mathrm{e}^{-\frac{1}{\sqrt{2}} \phi_{2}} \mathcal{F}_{\mu \nu}\right) \\
& \left.-\frac{1}{4}\left(4 m_{1}+m_{2}\right) \mathrm{e}^{\frac{1}{\sqrt{2}} \phi_{2}-\frac{1}{2 \sqrt{2}} \phi_{1}} \gamma_{7}\right] \epsilon_{i}-\frac{\mathrm{i}}{8 \sqrt{2}} \mathrm{e}^{\frac{1}{2 \sqrt{2}} \phi_{1}} \gamma^{\mu \nu} F_{\mu \nu i}{ }^{j} \epsilon_{j}, \\
& \delta \chi_{i}=\left[-\frac{1}{2 \sqrt{2}} \gamma^{\mu} \partial_{\mu} \phi_{2}+\frac{1}{2}\left(m_{1}-m_{2}\right) \mathcal{A}_{\mu} \gamma^{\mu}-\frac{1}{2}\left(m_{1}-m_{2}\right) \mathrm{e}^{\frac{1}{\sqrt{2}} \phi_{2}-\frac{1}{2 \sqrt{2}} \phi_{1}} \gamma_{7}\right. \\
& \left.-\frac{1}{8} \mathrm{e}^{\frac{1}{2 \sqrt{2}} \phi_{1}} \gamma^{\mu \nu} \gamma_{7}\left(-\mathrm{e}^{\frac{1}{\sqrt{2}} \phi_{2}} H_{\mu \nu}+\mathrm{e}^{-\frac{1}{\sqrt{2}} \phi_{2}} \mathcal{F}_{\mu \nu}\right)\right] \epsilon_{i}-\frac{\mathrm{i}}{2 \sqrt{2}} \mathrm{e}^{\frac{1}{\sqrt{2}} \phi_{2}} \gamma^{\mu} \gamma_{7} Q_{\mu i}{ }^{j} \epsilon_{j} .
\end{aligned}
$$

Here the $D=6$ field strengths are given by

$$
\begin{aligned}
& H_{(3)}=\mathrm{d} B_{(2)}-\frac{1}{2} F_{(2)}^{a} \wedge A_{(1)}^{a}-\mathrm{d} B_{(1)} \wedge \mathcal{A}_{(1)}-2\left(m_{2}-m_{1}\right) B_{(2)} \wedge \mathcal{A}_{(1)}+\frac{1}{2} \Phi^{a} F_{(2)}^{a} \wedge \mathcal{A}_{(1)}, \\
& H_{(2)}=\mathrm{d} B_{(1)}-\frac{1}{2} \Phi^{a} F_{(2)}^{a}+\frac{1}{2} Q_{(1)}^{a} \wedge A_{(1)}^{a}-\frac{1}{2} \Phi^{a} Q_{(1)}^{a} \wedge \mathcal{A}_{(1)}+2\left(m_{2}-m_{1}\right) B_{(2)}, \\
& F_{(2)}^{a}=\mathrm{d} A_{(1)}^{a}-\mathrm{d} \Phi^{a} \wedge \mathcal{A}_{(1)}+\left(m_{2}-m_{1}\right) A_{(1)}^{a} \wedge \mathcal{A}_{(1)}, \\
& Q_{(1)}^{a}=\mathrm{d} \Phi^{a}-\left(m_{2}-m_{1}\right) A_{(1)}^{a},
\end{aligned}
$$

with $Q_{(1) i}^{j}=Q_{(1)}^{a}\left(-\tau^{a}\right)_{i j}$, etc. 
The gravitino transformation in (2.9) demonstrates that the $\mathrm{Sp}(1)$ singlet graviphoton arises as a linear combination of $H_{\mu \nu}$ and $\mathcal{F}_{\mu \nu}$. Note, further, that these transformations reduce to those of ordinary ungauged $\mathcal{N}=(1,1)$ supergravity coupled to a vector multiplet in the limit of vanishing $m_{1}$ and $m_{2}$.

\subsection{Generalized supersymmetry in six dimensions}

Given the bosonic (2.6) and fermionic (2.8) reductions, it is now a matter of substituting these expressions into (2.3) to obtain the $D=6$ bosonic transformations. We find

$$
\begin{aligned}
& \delta \phi_{1}=-\frac{1}{2 \sqrt{2}} \bar{\epsilon}^{i} \lambda_{i} \\
& \delta \phi_{2}=-\frac{1}{2 \sqrt{2}} \bar{\epsilon}^{i} \chi_{i} \\
& \delta g_{\mu \nu}=\frac{1}{2} \bar{\epsilon}^{i} \gamma_{(\mu} \psi_{\nu) i} \\
& \delta \mathcal{A}_{\mu}=\frac{1}{4} \mathrm{e}^{-\frac{1}{2 \sqrt{2}} \phi_{1}+\frac{1}{\sqrt{2}} \phi_{2}}\left[\bar{\epsilon}^{i} \gamma_{7}\left(\psi_{\mu i}+\frac{1}{2} \gamma_{\mu} \lambda_{i}\right)+\bar{\epsilon}^{i} \gamma_{\mu} \gamma_{7} \chi_{i}\right] \\
& \delta A_{\mu i}^{j}=-\Phi_{i}^{j} \delta \mathcal{A}_{\mu}-\frac{\mathrm{i}}{\sqrt{2}} \mathrm{e}^{-\frac{1}{2 \sqrt{2}} \phi_{1}} \bar{\epsilon}^{j}\left(\psi_{\mu i}+\frac{1}{2} \gamma_{\mu} \lambda_{i}\right), \\
& \delta \Phi_{i}{ }^{j}=-\frac{\mathrm{i}}{\sqrt{2}} \mathrm{e}^{-\frac{1}{\sqrt{2}} \phi_{2}} \bar{\chi}^{j} \gamma_{7} \epsilon_{i}, \\
& \delta B_{\mu}=\frac{1}{4} \Phi_{i}{ }^{j}\left(\delta A_{\mu j}{ }^{i}+\Phi_{j}{ }^{i} \delta \mathcal{A}_{\mu}\right)-\frac{1}{4} A_{\mu i}{ }^{j} \delta \Phi_{j}{ }^{i} \\
& +\frac{1}{4} \mathrm{e}^{-\frac{1}{2 \sqrt{2}} \phi_{1}-\frac{1}{\sqrt{2}} \phi_{2}}\left[\bar{\epsilon}^{i} \gamma_{7}\left(\psi_{\mu i}+\frac{1}{2} \gamma_{\mu} \lambda_{i}\right)-\bar{\epsilon}^{i} \gamma_{\mu} \gamma_{7} \chi_{i}\right] \\
& \delta B_{\mu \nu}=-\frac{1}{2} A_{[\mu i}{ }^{j} \Phi_{j}{ }^{i} \delta \mathcal{A}_{\nu]}-2 B_{[\mu} \delta \mathcal{A}_{\nu]}-\frac{1}{2} A_{[\mu i}{ }^{j} \delta A_{\nu] j}{ }^{i}-\frac{1}{2} \mathrm{e}^{-\frac{1}{\sqrt{2}} \phi_{1}} \bar{\epsilon}^{i}\left(\gamma_{[\mu} \psi_{\nu] i}+\frac{1}{2} \gamma_{\mu \nu} \lambda_{i}\right) .
\end{aligned}
$$

This result, combined with (2.9), yields the complete (lowest order) supersymmetry transformations of the variant $\mathcal{N}=(1,1)$ supergravity coupled to a vector multiplet. Note that in obtaining (2.9) and (2.11), it was crucial that the ansatz (2.8) allowed a consistent reduction from seven to six dimensions, in which the dependence on the $z$ coordinate cancelled in the seven-dimensional transformation rules. This guarantees that the resulting six-dimensional supersymmetry transformations are symmetries of the six-dimensional variant supergravity.

As noted in [6], the vector multiplet may be truncated away by setting $m_{1}=m_{2}$ as well as

$$
\phi_{2}=0, \quad \Phi_{i}^{j}=0, \quad B_{\mu}=\mathcal{A}_{\mu}=\frac{1}{\sqrt{2}} A_{\mu}, \quad \chi_{i}=0 .
$$

In this case, the $D=6$ field strengths of (2.10) simplify to

$$
H_{(3)}=\mathrm{d} B_{(2)}-\frac{1}{2} F_{(2)}^{a} \wedge A_{(1)}^{a}-\frac{1}{2} F_{(2)} \wedge A_{(1)}, \quad F_{(2)}=\mathrm{d} A_{(1)}, \quad F_{(2)}^{a}=\mathrm{d} A_{(1)}^{a} .
$$

The resulting six-dimensional theory has field content $\left(g_{\mu \nu}, A_{\mu}, A_{\mu}^{a}, B_{\mu \nu}, \phi_{1}, \psi_{\mu i}, \lambda_{i}\right)$ and supersymmetry transformations 


$$
\begin{aligned}
& \delta \psi_{\mu i}=\left[\nabla_{\mu}-\frac{5}{8 \sqrt{2}} m \gamma_{\mu} \gamma^{\nu} A_{\nu}-\frac{1}{48} \mathrm{e}^{\frac{1}{\sqrt{2}} \phi_{1}}\left(\gamma_{\mu}^{\nu \rho \sigma}-3 \delta_{\mu}^{\nu} \gamma^{\rho \sigma}\right) H_{\nu \rho \sigma}\right. \\
& \left.-\frac{1}{16 \sqrt{2}} \mathrm{e}^{\frac{1}{2 \sqrt{2}} \phi_{1}}\left(\gamma_{\mu}^{\nu \rho}-6 \delta_{\mu}^{\nu} \gamma^{\rho}\right) \gamma_{7} F_{\nu \rho}+\frac{5}{8} m \mathrm{e}^{-\frac{1}{2 \sqrt{2}} \phi_{1}} \gamma_{\mu} \gamma_{7}\right] \epsilon_{i} \\
& +\frac{\mathrm{i}}{16 \sqrt{2}} \mathrm{e}^{\frac{1}{2 \sqrt{2}} \phi_{1}}\left(\gamma_{\mu}^{\nu \rho}-6 \delta_{\mu}^{\nu} \gamma^{\rho}\right) F_{\nu \rho i}{ }^{j} \epsilon_{j}, \\
& \delta \lambda_{i}=\left[-\frac{1}{2 \sqrt{2}} \gamma^{\mu} \partial_{\mu} \phi_{1}+\frac{5}{4 \sqrt{2}} m A_{\mu} \gamma^{\mu}+\frac{1}{24} \mathrm{e}^{\frac{1}{\sqrt{2}} \phi_{1}} H_{\mu \nu \rho} \gamma^{\mu \nu \rho}\right. \\
& \left.+\frac{1}{8 \sqrt{2}} \mathrm{e}^{\frac{1}{2 \sqrt{2}} \phi_{1}} \gamma^{\mu \nu} \gamma_{7} F_{\mu \nu}-\frac{5}{4} m \mathrm{e}^{-\frac{1}{2 \sqrt{2}} \phi_{1}} \gamma_{7}\right] \epsilon_{i}-\frac{\mathrm{i}}{8 \sqrt{2}} \mathrm{e}^{\frac{1}{2 \sqrt{2}} \phi_{1}} \gamma^{\mu \nu} F_{\mu \nu i}{ }^{j} \epsilon_{j}, \\
& \delta \phi_{1}=-\frac{1}{2 \sqrt{2}} \bar{\epsilon}^{i} \lambda_{i} \\
& \delta g_{\mu \nu}=\frac{1}{2} \bar{\epsilon}^{i} \gamma_{(\mu} \psi_{\nu) i} \\
& \delta A_{\mu}=\frac{1}{2 \sqrt{2}} \mathrm{e}^{-\frac{1}{2 \sqrt{2}} \phi_{1}} \bar{\epsilon}^{i} \gamma_{7}\left(\psi_{\mu i}+\frac{1}{2} \gamma_{\mu} \lambda_{i}\right), \\
& \delta A_{\mu i}{ }^{j}=-\frac{\mathrm{i}}{\sqrt{2}} \mathrm{e}^{-\frac{1}{2 \sqrt{2}} \phi_{1}} \bar{\epsilon}^{j}\left(\psi_{\mu i}+\frac{1}{2} \gamma_{\mu} \lambda_{i}\right), \\
& \delta B_{\mu \nu}=-A_{[\mu} \delta A_{\nu]}-\frac{1}{2} A_{[\mu i}^{j} \delta A_{\nu] j}^{i}-\frac{1}{2} \mathrm{e}^{-\frac{1}{\sqrt{2}} \phi_{1}} \bar{\epsilon}^{i}\left(\gamma_{[\mu} \psi_{\nu] i}+\frac{1}{2} \gamma_{\mu \nu} \lambda_{i}\right) .
\end{aligned}
$$

These transformations reduce to those of [9] when $m \rightarrow 0$.

On the other hand, for $m \neq 0$, the generalized reduction yields additional terms in $\delta \psi_{\mu i}$ and $\delta \lambda_{i}$. Furthermore, these $m$-dependent terms do not have the usual structure for a gauged supergravity. In particular, the gauge potential $A_{(1)}$ does not appear in $\delta \psi_{\mu i}$ as a minimal coupling term $D_{\mu}=\nabla_{\mu}+\mathrm{i} g A_{\mu}$ to a charged spinor, yet shows up as a bare potential term in $\delta \lambda_{i}$. This is consistent with $A_{(1)}$ showing up as well in the bosonic equations of motion [6]. For this reason, it is natural to suspect that the local supersymmetry algebra satisfied by this theory is necessarily modified. To see this, we may examine, e.g., the double variation on $\phi_{1}$. We find

$$
\left[\delta_{1}, \delta_{2}\right] \phi_{1}=\frac{1}{4} \xi^{\mu} \partial_{\mu} \phi_{1}-\frac{5}{4 \sqrt{2}} m\left(\frac{1}{\sqrt{2}} \xi^{\mu} A_{\mu}-\mathrm{e}^{-\frac{1}{2 \sqrt{2}} \phi_{1}}\left(\bar{\epsilon}_{2}^{i} \gamma_{7} \epsilon_{1 i}\right)\right),
$$

where $\xi^{\mu}=\bar{\epsilon}_{2}^{i} \gamma^{\mu} \epsilon_{1 i}$. The additional terms vanish when $m=0$.

\subsection{String frame formalism}

Some of the aspects of this theory become simpler to discuss in the string frame. The relation between the quantities in the Einstein frame and string frame is given by

$$
\begin{aligned}
& g_{\mu \nu}=\mathrm{e}^{-\phi} \tilde{g}_{\mu \nu}, \quad F_{(2)}^{a}=\widetilde{F}_{(2)}^{a}, \quad B_{(2)}=\widetilde{B}_{(2)}, \quad \mathrm{d} \phi+\frac{5}{2 \sqrt{2}} m A_{(1)}=\widetilde{B}_{(1)}, \\
& F_{(2)}=\widetilde{F}_{(2)}, \quad \phi_{1}=-\sqrt{2} \phi, \quad \epsilon=\mathrm{e}^{-\frac{1}{4} \phi} \tilde{\epsilon}, \quad \lambda=\mathrm{e}^{\frac{1}{4} \phi} \tilde{\lambda}, \quad \psi_{\mu}=\mathrm{e}^{-\frac{1}{4} \phi} \tilde{\psi}_{\mu},
\end{aligned}
$$

where the tilded variables are those in the string frame. The bosonic equations of motion are then given by 


$$
\begin{aligned}
& \widetilde{\nabla}^{\sigma} \widetilde{H}_{\mu \nu \sigma}=2 \widetilde{H}_{\mu \nu \sigma} \widetilde{B}^{\sigma}-\frac{5}{\sqrt{2}} m \widetilde{F}_{\mu \nu}, \\
& \widetilde{\nabla}^{v} \widetilde{F}_{\mu \nu}=2 \widetilde{F}_{\mu \nu} \widetilde{B}^{v}+\frac{1}{2} \widetilde{H}_{\mu \nu \sigma} \widetilde{F}^{\nu \sigma}, \\
& \widetilde{\nabla}^{v} \widetilde{F}_{\mu \nu}^{a}=2 \widetilde{F}_{\mu \nu}^{a} \widetilde{B}^{\nu}+\frac{1}{2} \widetilde{H}_{\mu \nu \sigma} \widetilde{F}^{a v \sigma}, \\
& \widetilde{\nabla}^{\mu} \widetilde{B}_{\mu}=2 \widetilde{B}_{(1)}^{2}-\frac{1}{12} \widetilde{H}_{(3)}^{2}-\frac{1}{8}\left(\widetilde{F}_{(2)}^{2}+\left(\widetilde{F}_{(2)}^{a}\right)^{2}\right)+\frac{25}{2} m^{2}, \\
& \widetilde{R}_{\mu \nu}=-\widetilde{\nabla}_{\mu} \widetilde{B}_{\nu}-\widetilde{\nabla}_{\nu} \widetilde{B}_{\mu}+\frac{1}{4} \widetilde{H}_{\mu \sigma \rho} \widetilde{H}_{\nu}{ }^{\sigma \rho}+\frac{1}{2}\left(\widetilde{F}_{\mu \rho} \widetilde{F}_{\nu}{ }^{\rho}+\widetilde{F}_{\mu \rho}^{a} \widetilde{F}_{\nu}^{a \rho}\right) .
\end{aligned}
$$

Thus we see that the dilaton $\phi_{1}$ is eliminated by the gauge field $A_{(1)}$ to give rise to a massive vector $\widetilde{B}_{(1)}$ field. The supersymmetric transformation rules for the fermions are given by

$$
\begin{aligned}
& \delta \tilde{\lambda}_{i}= {\left[\frac{1}{2} \widetilde{B}_{\mu} \tilde{\gamma}^{\mu}+\frac{1}{24} \widetilde{H}_{\mu \nu \rho} \tilde{\gamma}^{\mu \nu \rho}+\frac{1}{8 \sqrt{2}} \tilde{\gamma}^{\mu \nu} \gamma_{7} \widetilde{F}_{\mu \nu}-\frac{5}{4} m \gamma_{7}\right] \tilde{\epsilon}_{i}-\frac{\mathrm{i}}{8 \sqrt{2}} \tilde{\gamma}^{\mu \nu} \widetilde{F}_{\mu \nu i}{ }^{j} \tilde{\epsilon}_{j}, } \\
& \delta \tilde{\psi}_{\mu i}=\left[\widetilde{\nabla}_{\mu}-\frac{1}{4} \widetilde{B}_{\nu} \tilde{\gamma}_{\mu} \tilde{\gamma}^{\nu}-\frac{1}{48}\left(\tilde{\gamma}_{\mu}^{\nu \rho \sigma}-3 \delta_{\mu}^{\nu} \tilde{\gamma}^{\rho \sigma}\right) \widetilde{H}_{\nu \rho \sigma}+\frac{5}{8} m \tilde{\gamma}_{\mu} \gamma_{7}\right. \\
&\left.\quad-\frac{1}{16 \sqrt{2}}\left(\tilde{\gamma}_{\mu}^{\nu \rho}-6 \delta_{\mu}^{\nu} \tilde{\gamma}^{\rho}\right) \gamma_{7} \widetilde{F}_{\nu \rho}\right] \tilde{\epsilon}_{i}+\frac{\mathrm{i}}{16 \sqrt{2}}\left(\tilde{\gamma}_{\mu}^{\nu \rho}-6 \delta_{\mu}^{\nu} \tilde{\gamma}^{\rho}\right) \widetilde{F}_{\nu \rho i}^{j} \tilde{\epsilon}_{j} .
\end{aligned}
$$

It is of interest to note that the supersymmetric transformation rule for the shifted gravitino, $\widetilde{\widetilde{\psi}}_{\mu}=\widetilde{\psi}_{\mu}+\frac{1}{2} \tilde{\gamma}_{\mu} \tilde{\lambda}$, given by

$$
\delta \widetilde{\psi}_{\mu i}=\left[\widetilde{\nabla}_{\mu}+\frac{1}{8} \widetilde{H}_{\mu \nu \rho} \tilde{\gamma}^{\nu \rho}+\frac{1}{2 \sqrt{2}} \tilde{\gamma}^{v} \gamma_{7} \widetilde{F}_{\mu \nu}\right] \tilde{\epsilon}_{i}-\frac{\mathrm{i}}{2 \sqrt{2}} \tilde{\gamma}^{\nu} \widetilde{F}_{\mu \nu i}^{j} \tilde{\epsilon}_{j},
$$

does not depend on $m$.

\section{The (Minkowski) $)_{4} \times S^{2}$ reduction}

The $D=6$ theory obtained in [6] does not admit a Lagrangian formulation since the bare potential $A_{(1)}$ appears directly in the equations of motion. This is also apparent from the supersymmetry variations obtained in the previous section. However, for field configurations with vanishing $A_{(1)}$, the resulting bosonic equations of motion may be obtained from the Lagrangian

$$
\mathcal{L}=\hat{R} \hat{*} \mathbb{1}-\frac{1}{4} \hat{*} \mathrm{~d} \hat{\phi} \wedge \mathrm{d} \hat{\phi}-\frac{1}{2} \mathrm{e}^{\hat{\phi}_{\hat{*}}} \hat{H}_{(3)} \wedge \hat{H}_{(3)}-\frac{1}{2} \mathrm{e}^{\frac{1}{2} \hat{\phi}} \hat{*} \hat{F}_{(2)}^{a} \wedge \hat{F}_{(2)}^{a}-8 g^{2} \mathrm{e}^{-\frac{1}{2} \hat{\phi}} \hat{*} \mathbb{1} .
$$

We have now introduced carets to denote six-dimensional fields, in anticipation of a subsequent reduction to four dimensions. Furthermore, we have defined $\hat{\phi}=\sqrt{2} \phi_{1}$ to simplify the subsequent expressions and have defined $5 m=2 \sqrt{2} g$.

Curiously, this bosonic Lagrangian is identical to that of the Salam-Sezgin model, with the exception that there are three vector fields instead of one. As a result, this model clearly admits a bosonic $M_{4} \times S^{2}$ reduction, where $M_{4}$ denotes four-dimensional Minkowski spacetime. On the other hand, the supersymmetry of the reduction must still be verified, as the supersymmetry transformations of the variant $\mathcal{N}=(1,1)$ theory differ from that of the gauged $\mathcal{N}=(1,0)$ model.

In order to investigate the supersymmetry, it is useful to rewrite the six-dimensional symplectic-Majorana spinors using Dirac notation. A symplectic-Majorana spinor satisfies the reality condition $\left(\hat{\psi}_{i}\right)^{*}=-\epsilon^{i j} \hat{C} \hat{\gamma}_{0} \hat{\psi}_{j}$, where the charge conjugation matrix $\hat{C}$ satisfies $\hat{C}^{T}=\hat{C}$ and $\hat{C}^{\dagger} \hat{C}=1$. We may now form the Dirac combination $\hat{\psi}=\hat{\psi}_{1}+\mathrm{i} \hat{\psi}_{2}$, with complex conjugate $\hat{\psi}^{*}=-\mathrm{i} \hat{C} \hat{\gamma}_{0}\left(\hat{\psi}_{1}-\mathrm{i} \hat{\psi}_{2}\right)$. Equivalently, these definitions may be inverted to yield

$$
\hat{\psi}_{1}=\frac{1}{2}\left(\hat{\psi}-\mathrm{i} \hat{\gamma}_{0} \hat{C}^{*} \hat{\psi}^{*}\right), \quad \hat{\psi}_{2}=\frac{1}{2 i}\left(\hat{\psi}+\mathrm{i} \hat{\gamma}_{0} \hat{C}^{*} \hat{\psi}^{*}\right)
$$


As a result, for $\hat{A}_{(1)}=0$, the supersymmetry transformations (2.14) may be rewritten as

$$
\begin{gathered}
\delta \hat{\psi}_{\mu}=\left[\hat{\nabla}_{\mu}-\frac{1}{48} \mathrm{e}^{\frac{1}{2} \hat{\phi}}\left(\hat{\gamma}_{\mu}^{\nu \rho \sigma}-3 \delta_{\mu}^{\nu} \hat{\gamma}^{\rho \sigma}\right) \hat{H}_{\nu \rho \sigma}+\frac{1}{2 \sqrt{2}} g \mathrm{e}^{-\frac{1}{4} \hat{\phi}} \hat{\gamma}_{\mu} \hat{\gamma}_{7}\right] \hat{\epsilon} \\
+\frac{\mathrm{i}}{16 \sqrt{2}} \mathrm{e}^{\frac{1}{4} \hat{\phi}}\left(\hat{\gamma}_{\mu}^{\nu \rho}-6 \delta_{\mu}^{\nu} \hat{\gamma}^{\rho}\right)\left(\hat{F}_{\nu \rho}^{2} \hat{\epsilon}-\left(\hat{F}_{\nu \rho}^{1}-\mathrm{i} \hat{F}_{\nu \rho}^{3}\right) \hat{\gamma}_{0} \hat{C}^{*} \hat{\epsilon}^{*}\right), \\
\delta \hat{\lambda}=\left[-\frac{1}{4} \hat{\gamma}^{\mu} \partial_{\mu} \hat{\phi}+\frac{1}{24} \mathrm{e}^{\frac{1}{2} \hat{\phi}} \hat{H}_{\mu \nu \rho} \hat{\gamma}^{\mu \nu \rho}-\frac{1}{\sqrt{2}} g \mathrm{e}^{-\frac{1}{4} \hat{\phi}} \hat{\gamma}_{7}\right] \hat{\epsilon} \\
-\frac{\mathrm{i}}{8 \sqrt{2}} \mathrm{e}^{\frac{1}{4} \hat{\phi}} \hat{\gamma}^{\mu \nu}\left(\hat{F}_{\mu \nu}^{2} \hat{\epsilon}-\left(\hat{F}_{\mu \nu}^{1}-\mathrm{i} \hat{F}_{\mu \nu}^{3}\right) \hat{\gamma}_{0} \hat{C}^{*} \hat{\epsilon}^{*}\right),
\end{gathered}
$$

for the fermions and

$$
\begin{aligned}
& \delta \hat{\phi}=-\frac{1}{4}[\overline{\hat{\epsilon}} \hat{\lambda}+\overline{\hat{\lambda}} \hat{\epsilon}] \\
& \delta \hat{g}_{\mu \nu}=\frac{1}{2}\left[\overline{\hat{\epsilon}} \hat{\gamma}_{(\mu} \hat{\psi}_{\nu)}-\overline{\hat{\psi}}_{(\mu} \hat{\gamma}_{\nu)} \hat{\epsilon}\right] \\
& \delta \hat{A}_{\mu}=\frac{1}{4 \sqrt{2}} \mathrm{e}^{-\frac{1}{4} \hat{\phi}}\left[\overline{\hat{\epsilon}} \hat{\gamma}_{7}\left(\hat{\psi}_{\mu}+\frac{1}{2} \hat{\gamma}_{\mu} \hat{\lambda}\right)-\left(\overline{\hat{\psi}}_{\mu}-\frac{1}{2} \overline{\hat{\lambda}}_{\mu}\right) \hat{\gamma}_{7} \hat{\epsilon}\right] \\
& \delta \hat{A}_{\mu}^{1}=-\frac{1}{2 \sqrt{2}} \mathrm{e}^{-\frac{1}{4} \hat{\phi}} \operatorname{Im}\left[\hat{\epsilon}^{T} \hat{C}\left(\hat{\psi}_{\mu}+\frac{1}{2} \hat{\gamma}_{\mu} \hat{\lambda}\right)\right], \\
& \delta \hat{A}_{\mu}^{2}=-\frac{\mathrm{i}}{4 \sqrt{2}} \mathrm{e}^{-\frac{1}{4} \hat{\phi}}\left[\overline{\hat{\epsilon}}\left(\hat{\psi}_{\mu}+\frac{1}{2} \hat{\gamma}_{\mu} \hat{\lambda}\right)-\left(\overline{\hat{\psi}}_{\mu}-\frac{1}{2} \overline{\hat{\lambda}}_{\mu}\right) \hat{\epsilon}\right], \\
& \delta \hat{A}_{\mu}^{3}=-\frac{1}{2 \sqrt{2}} \mathrm{e}^{-\frac{1}{4} \hat{\phi}} \operatorname{Re}\left[\hat{\epsilon}^{T} \hat{C}\left(\hat{\psi}_{\mu}+\frac{1}{2} \hat{\gamma}_{\mu} \hat{\lambda}\right)\right], \\
& \delta \hat{B}_{\mu \nu}=-\hat{A}_{[\mu}^{a} \delta \hat{A}_{\nu]}^{a}-\frac{1}{4} \mathrm{e}^{-\frac{1}{2} \hat{\phi}}\left[\overline{\hat{\epsilon}}\left(\hat{\gamma}_{[\mu} \hat{\psi}_{\nu]}+\frac{1}{2} \hat{\gamma}_{\mu \nu} \hat{\lambda}\right)+\left(\overline{\hat{\psi}}_{[\mu} \hat{\gamma}_{\nu]}-\frac{1}{2} \overline{\hat{\lambda}}_{\mu \nu}\right) \hat{\epsilon}\right],
\end{aligned}
$$

for the bosons. While we have set $\hat{A}_{\mu}=0$, it is important to retain its supersymmetry variation so that it is possible to check later for consistency. These expressions serve as the starting point for the subsequent analysis.

\subsection{Supersymmetry of the $M_{4} \times S^{2}$ vacuum}

The bosonic theory, given by (3.1), admits an $M_{4} \times S^{2}$ solution given by

$$
\mathrm{d} \hat{s}_{6}^{2}=\eta_{\mu \nu} \mathrm{d} x^{\mu} \mathrm{d} x^{\nu}+\frac{1}{8 g^{2}} \mathrm{~d} \Omega_{2}^{2}, \quad \hat{F}_{(2)}^{2}=\frac{1}{2 g} \Omega_{(2)},
$$

where $\Omega_{(2)}=\sin \theta \mathrm{d} \theta \wedge \mathrm{d} \varphi$ is the volume form on the unit $S^{2}$. Note that we have singled out the 2-component of the $\operatorname{Sp}(1)$ triplet gauge fields for convenience. While this choice is a natural one corresponding to the Dirac combination in (3.3), any other choice would yield the same result.

To examine the supersymmetry of the vacuum, we insert (3.5) into (3.3) to obtain

$$
\begin{aligned}
& \delta \hat{\psi}_{\alpha}=\left[\partial_{\alpha}+\frac{1}{\sqrt{2}} g \hat{\gamma}_{\alpha} \hat{\gamma}_{7} P_{+}\right] \hat{\epsilon}, \\
& \delta \hat{\psi}_{a}=\left[\nabla_{a}-\mathrm{i} \sqrt{2} g \hat{\gamma}_{a} \hat{\gamma}_{45}\right] \hat{\epsilon}+\frac{1}{\sqrt{2}} g \hat{\gamma}_{a} \hat{\gamma}_{7} P_{+} \hat{\epsilon}, \\
& \delta \hat{\lambda}=-\sqrt{2} g \hat{\gamma}_{7} P_{+} \hat{\epsilon},
\end{aligned}
$$


where $P_{ \pm}=\frac{1}{2}\left(1 \pm \mathrm{i} \hat{\gamma}^{45} \hat{\gamma}_{7}\right)$ is a half-BPS projection. These equations vanish for $\hat{\epsilon}=P_{-} \hat{\epsilon}_{0}$ where $\hat{\epsilon}_{0}$ solves the Killing spinor equation on the round 2-sphere, $\left[\nabla_{a}-\mathrm{i} \sqrt{2} g \hat{\gamma}_{a} \hat{\gamma}_{45}\right] \hat{\epsilon}_{0}=0$.

To be more precise, we decompose the six-dimensional Dirac matrices according to

$$
\begin{aligned}
& \hat{\gamma}_{\alpha}=\gamma_{\alpha} \otimes \sigma_{3}, \quad \hat{\gamma}_{4}=\mathbb{1} \otimes \sigma_{1}, \quad \hat{\gamma}_{5}=\mathbb{1} \otimes \sigma_{2}, \\
& \hat{\gamma}_{7}=\hat{\gamma}_{0} \hat{\gamma}_{1} \cdots \hat{\gamma}_{5}=\gamma^{5} \otimes \sigma_{3}, \quad \hat{C}=C \otimes \sigma_{2},
\end{aligned}
$$

where $C$ is now the four-dimensional charge conjugation matrix and $\gamma^{5}=\mathrm{i} \gamma_{0} \gamma_{1} \gamma_{2} \gamma_{3}$. Six-dimensional spinors $\hat{\epsilon}$ may then be written in terms of $M_{4}$ and $S^{2}$ spinors as $\hat{\epsilon}=\sum_{I} \epsilon_{I} \otimes \eta_{I}$ where $\eta_{I}$ is taken to be commuting. In this case, the Killing spinor equation on $S^{2}$ becomes $\left[\nabla_{a}+\sqrt{2} g \sigma_{a} \sigma_{3}\right] \eta_{I}=0$, and yields two independent solutions. Corresponding to the above choice of Dirac matrices, we find that in the basis $e^{4}=(2 \sqrt{2} g)^{-1} \mathrm{~d} \theta, e^{5}=(2 \sqrt{2} g)^{-1} \sin \theta \mathrm{d} \varphi$, the two independent Killing spinors can be written as

$$
\eta_{1}=\left(\begin{array}{c}
\cos \frac{1}{2} \theta \\
-\sin \frac{1}{2} \theta
\end{array}\right) \mathrm{e}^{\frac{1}{2} \varphi}, \quad \eta_{2}=\left(\begin{array}{c}
\sin \frac{1}{2} \theta \\
\cos \frac{1}{2} \theta
\end{array}\right) \mathrm{e}^{-\frac{1}{2} \varphi} .
$$

It is easily seen that these satisfy the conditions

$$
\bar{\eta}_{I} \eta_{J}=\delta_{I J}, \quad \eta_{I}^{T} \sigma^{2} \eta_{J}=-\mathrm{i} \epsilon_{I J}, \quad \eta_{I}^{*}=\mathrm{i} \sigma^{2} \epsilon_{I J} \eta_{J} .
$$

Note that $\bar{\eta}_{I} \equiv \eta_{I}^{\dagger}$. Using the decomposition (3.7), the half-BPS projection operator takes the form $P_{ \pm}=\frac{1}{2}\left(1 \mp \gamma_{5}\right)$. As a result, the Killing spinors in the $M_{4} \times S^{2}$ background are given by

$$
\hat{\epsilon}=\epsilon_{I} \otimes \eta_{I} \quad\left(\epsilon_{I}=\gamma_{5} \epsilon_{I}\right)
$$

where the $\epsilon_{I}$ are a pair of constant $D=4$ Weyl spinors.

\subsection{Reduction to $D=4, \mathcal{N}=2$ supergravity}

The existence of a supersymmetric vacuum suggests that a consistent Kaluza-Klein reduction on $S^{2}$ is possible, yielding a Poincaré theory in four dimensions. Since the six-dimensional $\mathcal{N}=(1,1)$ theory has 16 real supersymmetries, and the vacuum breaks exactly half of them, the resulting theory corresponds to $\mathcal{N}=2$ supersymmetry in four dimensions.

The basic $\mathcal{N}=2$ supergravity multiplet consists of a graviton $g_{\mu \nu}$, graviphoton $A_{(1)}$ and a pair of Majorana gravitinos $\psi_{\mu i}$. In addition, $\mathcal{N}=2$ vector multiplets are given by a vector $A_{(1)}$, two real scalars $\phi$ and $a$, and a pair of Majorana gauginos $\chi_{i}$. We find that the six-dimensional field content reduces to yield $\mathcal{N}=2$ supergravity coupled to a single vector multiplet. The reduction ansatz for the bosons is given by

$$
\begin{aligned}
& \mathrm{d} \hat{s}_{6}^{2}=\mathrm{e}^{\frac{1}{2} \phi} \mathrm{d} s_{4}^{2}+\frac{1}{8 g^{2}} \mathrm{e}^{-\frac{1}{2} \phi} \mathrm{d} \Omega_{2}^{2}, \\
& \hat{F}_{(2)}^{2}=2 g \mathrm{e}^{\frac{1}{2} \phi} \epsilon_{a b} \hat{e}^{a} \wedge \hat{e}^{b}, \quad \hat{F}_{(2)}^{1}=F_{(2)}^{1}, \quad \hat{F}_{(2)}^{3}=F_{(2)}^{3}, \\
& \hat{H}_{(3)}=H_{(3)}, \quad \hat{\phi}=-\phi .
\end{aligned}
$$

Note that the graviphoton and matter vector field strengths are given by a combination of $F_{(2)}^{1}$ and $F_{(2)}^{3}$ (up to duality) as will be apparent below. The use of the 1- and 3-components of the $\mathrm{Sp}(1)$ triplet in the Kaluza-Klein reduction is dictated by the choice of turning on $F_{(2)}^{2}$ flux on the sphere.

It is straightforward to verify the consistency of the bosonic reduction. The resulting four-dimensional equations of motion may be obtained from the Lagrangian 
$\mathcal{L}=R * \mathbb{1}-\frac{1}{2} * \mathrm{~d} \phi \wedge \mathrm{d} \phi-\frac{1}{2} \mathrm{e}^{-2 \phi} * H_{(3)} \wedge H_{(3)}-\frac{1}{2} \mathrm{e}^{-\phi}\left(* F_{(2)}^{1} \wedge F_{(2)}^{1}+* F_{(2)}^{3} \wedge F_{(2)}^{3}\right)$.

The fermion reduction ansatz may be obtained by substituting the bosonic fields (3.11) into the six-dimensional gravitino and dilatino transformations (3.3). Starting with the latter, we see that

$$
\begin{array}{r}
\delta \hat{\lambda}=\sqrt{2} g \mathrm{e}^{\frac{1}{4} \phi} P_{+} \otimes \sigma_{3} \hat{\epsilon}+\mathrm{e}^{-\frac{1}{4} \phi}\left[\frac{1}{4} \gamma^{\mu} \partial_{\mu} \phi+\frac{1}{24} \mathrm{e}^{-\phi} H_{\mu \nu \rho} \gamma^{\mu \nu \rho}\right] \otimes \sigma_{3} \hat{\epsilon} \\
-\frac{\mathrm{i}}{8 \sqrt{2}} \mathrm{e}^{-\frac{1}{4} \phi}\left[\mathrm{e}^{-\frac{1}{2} \phi}\left(F_{\mu \nu}^{1}-\mathrm{i} F_{\mu \nu}^{3}\right) \gamma^{\mu \nu} \gamma_{0} C^{*}\right] \otimes \sigma_{3} \sigma_{2} \hat{\epsilon}^{*} .
\end{array}
$$

The first term vanishes on chiral spinors $P_{+} \hat{\epsilon}=0$, while the remaining terms combine to yield the four-dimensional gaugino transformation.

Turning to the gravitino variation, as usual the $D=6$ variation splits into a $D=4$ gravitino term, $\delta \hat{\psi}_{\alpha}$, as well as two internal variations, $\delta \hat{\psi}_{a}$. Since the $S^{2}$ symmetry is unbroken by the bosonic ansatz, the two internal components of the gravitino variation are related by symmetry. In fact, provided $\hat{\epsilon}$ is decomposed in terms of Killing spinors on the sphere, the $\delta \hat{\psi}_{a}$ variation has identical content to that of $\delta \hat{\lambda}$. (This is not in general true, but holds in the present case.) As a result, we find the fermionic reduction ansatz to have the form

$$
\begin{aligned}
& \hat{\epsilon}=\mathrm{e}^{\frac{1}{8} \phi} \epsilon_{I} \otimes \eta_{I}, \quad \hat{\lambda}=\mathrm{e}^{-\frac{1}{8} \phi} \chi_{I} \otimes \sigma_{3} \eta_{I}, \\
& \hat{\psi}_{\alpha}=\mathrm{e}^{-\frac{1}{8} \phi}\left[\psi_{\alpha I}+\frac{1}{2} \gamma_{\alpha} \chi_{I}\right] \otimes \eta_{I}, \quad \hat{\psi}_{a}=\mathrm{e}^{-\frac{1}{8} \phi}\left(-\frac{1}{2} \chi_{I}\right) \otimes \sigma_{a} \sigma_{3} \eta_{I} .
\end{aligned}
$$

Inserting this ansatz into (3.13) as well as the gravitino variations yields the fourdimensional supersymmetry transformations

$\delta \chi_{I}=\left[\frac{1}{4} \gamma^{\mu} \partial_{\mu} \phi+\frac{1}{24} \mathrm{e}^{-\phi} H_{\mu \nu \rho} \gamma^{\mu \nu \rho}\right] \epsilon_{I}-\frac{1}{4 \sqrt{2}} \mathrm{e}^{-\frac{1}{2} \phi}\left(F_{\mu \nu}^{1}-\mathrm{i} F_{\mu \nu}^{3}\right) \gamma^{\mu \nu} \gamma_{0} C^{*} \epsilon_{I J} \epsilon_{J}^{*}$,

$\delta \psi_{\mu I}=\left[\nabla_{\mu}-\frac{1}{24} \mathrm{e}^{-\phi} \gamma_{\mu}^{\nu \rho \sigma} H_{\nu \rho \sigma}\right] \epsilon_{I}-\frac{1}{8 \sqrt{2}} \mathrm{e}^{-\frac{1}{2} \phi}\left(F_{v \rho}^{1}-\mathrm{i} F_{\nu \rho}^{3}\right) \gamma^{\nu \rho} \gamma_{\mu} \gamma_{0} C^{*} \epsilon_{I J} \epsilon_{J}^{*}$

To obtain this result, we had to make use of the $\eta_{I}^{*}$ relation in (3.9). At this stage, we note that the gauge fields may be dualized in four dimensions, so that $F_{\mu \nu} \gamma^{\mu \nu}=-\mathrm{i} * F_{\mu \nu} \gamma^{\mu \nu} \gamma_{5}$. Since the four-dimensional spinors are given in a Weyl basis

$$
P_{+} \epsilon_{I}=0, \quad P_{+} \psi_{\alpha I}=0, \quad P_{-} \chi_{I}=0,
$$

where $P_{ \pm}=\frac{1}{2}\left(1 \mp \gamma_{5}\right)$, the above supersymmetry variations may be rewritten as

$\delta \chi_{I}=\left[\frac{1}{4} \gamma^{\mu} \partial_{\mu} \phi+\frac{1}{24} \mathrm{e}^{-\phi} H_{\mu \nu \rho} \gamma^{\mu \nu \rho}\right] \epsilon_{I}-\frac{1}{4 \sqrt{2}} \mathrm{e}^{-\frac{1}{2} \phi}\left(F_{\mu \nu}^{1}+* F_{\mu \nu}^{3}\right) \gamma^{\mu \nu} \gamma_{0} C^{*} \epsilon_{I J} \epsilon_{J}^{*}$,

$\delta \psi_{\mu I}=\left[\nabla_{\mu}-\frac{1}{24} \mathrm{e}^{-\phi} \gamma_{\mu}{ }^{\nu \rho \sigma} H_{\nu \rho \sigma}\right] \epsilon_{I}-\frac{1}{8 \sqrt{2}} \mathrm{e}^{-\frac{1}{2} \phi}\left(F_{\nu \rho}^{1}-* F_{v \rho}^{3}\right) \gamma^{\nu \rho} \gamma_{\mu} \gamma_{0} C^{*} \epsilon_{I J} \epsilon_{J}^{*}$.

This highlights the nature of the $\mathcal{N}=2$ graviphoton, $F_{(2)}^{(\mathcal{N}=2)}=\mathrm{e}^{-\frac{1}{2} \phi} F_{(2)}^{1}+\mathrm{e}^{\frac{1}{2} \phi} \widetilde{F}_{(2)}^{3}$, where $\widetilde{F}_{(2)}^{3}=\mathrm{e}^{-\phi} * F_{(2)}^{3}$.

Having completed the fermion reduction and supersymmetry variations, we now turn to the reduction of the bosonic variations, (3.4). The six-dimensional dilaton variation $\delta \hat{\phi}$ readily yields $\delta \phi=\frac{1}{2} \bar{\epsilon}_{I} \chi_{I}$. Similarly, the four-dimensional components of $\delta \hat{g}_{\mu \nu}$ yield $\delta g_{\mu \nu}=\frac{1}{2} \bar{\epsilon}_{I} \gamma_{(\mu} \psi_{\nu) I}$, while the internal components reduce to give the identical $\delta \phi$ transformation. This is a result of setting the internal components of the six-dimensional gravitino equal to the dilatino in the reduction.

In general, one obtains non-trivial vector field variations from the mixed components of the metric, $\delta \hat{g}_{\mu i}$, as well as directly from $\delta \hat{A}_{\mu}$. However, these terms vanish identically due to the $P_{ \pm}$chiralities of the four-dimensional spinors. Likewise, $\delta \hat{A}_{\mu}^{2}$ vanishes for the same reason. On the other hand, the additional complex conjugation appearing in $\delta \hat{A}_{\mu}^{1}$ and $\delta \hat{A}_{\mu}^{3}$ 
prevents these transformations from vanishing. The resulting four-dimensional variations then have the form

$$
\begin{aligned}
& \delta g_{\mu \nu}=\frac{1}{4}\left[\bar{\epsilon}_{I} \gamma_{(\mu} \psi_{\nu) I}-\bar{\psi}_{(\mu I} \gamma_{\nu)} \epsilon_{I}\right] \\
& \delta \phi=\frac{1}{4}\left[\bar{\epsilon}_{I} \chi_{I}+\bar{\chi}_{I} \epsilon_{I}\right], \\
& \delta B_{\mu \nu}=-\frac{1}{4} \mathrm{e}^{\phi}\left[\bar{\epsilon}_{I} \gamma_{[\mu} \psi_{\nu] I}+\bar{\psi}_{[\mu I} \gamma_{\nu]} \epsilon_{I}+\bar{\epsilon}_{I} \gamma_{\mu \nu} \chi_{I}-\bar{\chi}_{I} \gamma_{\mu \nu} \epsilon_{I}\right], \\
& \delta A_{\mu}^{1}=\frac{1}{2 \sqrt{2}} \mathrm{e}^{\frac{1}{2} \phi} \epsilon_{I J} \operatorname{Re}\left[\epsilon_{I}^{T} C\left(\psi_{\mu J}+\frac{1}{2} \gamma_{\mu} \chi_{J}\right)\right], \\
& \delta A_{\mu}^{3}=-\frac{1}{2 \sqrt{2}} \mathrm{e}^{\frac{1}{2} \phi} \epsilon_{I J} \operatorname{Im}\left[\epsilon_{I}^{T} C\left(\psi_{\mu J}+\frac{1}{2} \gamma_{\mu} \chi_{J}\right)\right] .
\end{aligned}
$$

We have verified that all variations of fields initially set to zero vanish, either identically or through four-dimensional chirality. This verifies the consistency of the supersymmetric reduction to $\mathcal{N}=2$ supergravity coupled to a single vector multiplet.

\subsection{Truncation to $D=4, \mathcal{N}=1$ supergravity}

While we have retained $\mathcal{N}=2$ supersymmetry in the above reduction, there is a natural truncation to $\mathcal{N}=1$. This may be accomplished by removing one of the two supersymmetry parameters by setting $\epsilon_{I}=\hat{n}_{I} \epsilon$ where $\hat{n}_{I}$ is any constant unit vector. At the same time, it is necessary to truncate the $\mathcal{N}=1$ gravitino and vector multiplets, leaving $\mathcal{N}=1$ supergravity coupled to a chiral multiplet. In the bosonic sector, this corresponds to setting $A_{\mu}^{1}=A_{\mu}^{3}=0$. The resulting bosonic Lagrangian is given by

$$
\mathcal{L}=R * \mathbb{1}-\frac{1}{2} * \mathrm{~d} \phi \wedge \mathrm{d} \phi-\frac{1}{2} \mathrm{e}^{-2 \phi} * H_{(3)} \wedge H_{(3)},
$$

while the relevant supersymmetry transformations are

$$
\begin{aligned}
& \delta \chi=\left[\frac{1}{4} \gamma^{\mu} \partial_{\mu} \phi+\frac{1}{24} \mathrm{e}^{-\phi} H_{\mu \nu \rho} \gamma^{\mu \nu \rho}\right] \epsilon, \\
& \delta \psi_{\mu}=\left[\nabla_{\mu}-\frac{1}{24} \mathrm{e}^{-\phi} \gamma_{\mu}^{\nu \rho \sigma} H_{\nu \rho \sigma}\right] \epsilon, \\
& \delta g_{\mu \nu}=\frac{1}{4}\left[\bar{\epsilon} \gamma_{(\mu} \psi_{\nu)}-\bar{\psi}\left(\mu \gamma_{\nu)} \epsilon\right],\right. \\
& \delta \phi=\frac{1}{4}[\bar{\epsilon} \chi+\bar{\chi} \epsilon], \\
& \delta B_{\mu \nu}=-\frac{1}{4} \mathrm{e}^{\phi}\left[\bar{\epsilon} \gamma_{[\mu} \psi_{\nu]}+\bar{\psi}_{[\mu} \gamma_{\nu]} \epsilon+\bar{\epsilon} \gamma_{\mu \nu} \chi-\bar{\chi} \gamma_{\mu \nu} \epsilon\right] .
\end{aligned}
$$

\section{BPS solutions}

The bosonic Lagrangian (3.12) admits a dyonic black-hole solution where $F_{(2)}^{1}$ is electric and $F_{(2)}^{3}$ is magnetic (or vice versa). The solution is given by

$$
\begin{aligned}
& \mathrm{d} s_{4}^{2}=-\left(\mathcal{H}_{1} \mathcal{H}_{3}\right)^{-1} \mathrm{~d} t^{2}+\mathcal{H}_{1} \mathcal{H}_{3}\left(\mathrm{~d} r^{2}+r^{2} \mathrm{~d} \widetilde{\Omega}_{2}^{2}\right), \\
& F_{(2)}^{1}=\mathrm{d} t \wedge \mathrm{d} \mathcal{H}_{1}^{-1}, \quad F_{(2)}^{3}=q_{3} \widetilde{\Omega}_{(2)}, \\
& \phi=-\log \left(\mathcal{H}_{1} / \mathcal{H}_{3}\right),
\end{aligned}
$$

where $\mathcal{H}_{1}=1+q_{1} / r$ and $\mathcal{H}_{3}=1+q_{3} / r$ are two harmonic functions in the Euclidean threedimensional transverse space. It becomes the standard Reissner-Nordström black hole when $\mathcal{H}_{1}=\mathcal{H}_{3}$. We can easily lift the solution back to $D=6$ dimensions, and it becomes 


$$
\begin{aligned}
& \mathrm{d} s_{6}^{2}=\left(\mathcal{H}_{1} / \mathcal{H}_{3}\right)^{\frac{1}{2}}\left[-\mathcal{H}_{1}^{-2} \mathrm{~d} t^{2}+\mathcal{H}_{3}^{2}\left(\mathrm{~d} r^{2}+r^{2} \mathrm{~d} \widetilde{\Omega}_{2}^{2}\right)+\frac{1}{8 g^{2}} \mathrm{~d} \Omega_{2}^{2}\right], \\
& \hat{F}_{(2)}^{2}=\frac{1}{2} g^{-1} \Omega_{(2)}, \quad \hat{F}_{(2)}^{1}=\mathrm{d} t \wedge \mathrm{d} \mathcal{H}_{1}^{-1}, \quad \hat{F}_{(2)}^{3}=q_{3} \widetilde{\Omega}_{(2)}, \\
& \hat{\phi}=\log \left(\mathcal{H}_{1} / \mathcal{H}_{3}\right) .
\end{aligned}
$$

In the near-horizon limit, the geometry becomes $\operatorname{AdS}{ }_{2} \times S^{2} \times S^{2}$. For $\mathcal{H}_{1}=\mathcal{H}_{3}$, the metric is the direct product of an $S^{2}$ and the Reissner-Nordström black hole. In the string frame, the metric is given by

$$
\mathrm{d} s_{\text {str }}^{2}=-\mathcal{H}_{1}^{-2} \mathrm{~d} t^{2}+\mathcal{H}_{3}^{2}\left(\mathrm{~d} r^{2}+r^{2} \mathrm{~d} \widetilde{\Omega}_{2}^{2}\right)+\frac{1}{8 g^{2}} \mathrm{~d} \Omega_{2}^{2} .
$$

\section{5. (Minkowski $)_{3} \times S^{3}$ vacuum}

The variant $\mathcal{N}=(1,1)$ six-dimensional supergravity has the unusual feature that it admits not only a supersymmetric (Minkowski) ${ }_{4} \times S^{2}$ vacuum, but also a supersymmetric (Minkowski) $)_{3} \times S^{3}$ vacuum. This is quite different from the situation in the Salam-Sezgin theory; although the Salam-Sezgin model admits a (Minkowski) $)_{3} \times S^{3}$ solution as well as a supersymmetric (Minkowski) $)_{4} \times S^{2}$ solution, the former is non-supersymmetric.

To construct the supersymmetric (Minkowski) $)_{3} \times S^{3}$ solution in the variant $\mathcal{N}=(1,1)$ supergravity, we make a standard Freund-Rubin type ansatz in which

$$
\mathrm{d} \hat{s}_{6}^{2}=\mathrm{d} x^{\mu} \mathrm{d} x_{\mu}+\mathrm{d} s_{3}^{2}, \quad \hat{H}_{(3)}=q \epsilon_{(3)}, \quad \hat{\phi}=0,
$$

where $\mathrm{d} s_{3}^{2}$ is the metric on a round $S^{3}$, with volume form $\epsilon_{(3)}$ and all other fields are set to zero. We find that this solves the six-dimensional equations of motion if

$$
q=2 \sqrt{2} g .
$$

The $S^{3}$ metric has the Ricci tensor given by $R_{i j}=4 g^{2} g_{i j}$.

To establish the supersymmetry of the solution, we decompose the six-dimensional Dirac matrices as

$$
\hat{\gamma}_{\mu}=\gamma_{\mu} \otimes \mathbb{1} \otimes \sigma_{2}, \quad \hat{\gamma}_{i}=\mathbb{1} \otimes \gamma_{i} \otimes \sigma_{1}, \quad \hat{\gamma}_{7}=\mathbb{1} \otimes \mathbb{1} \otimes \sigma_{3} .
$$

Writing $\hat{\epsilon}=\epsilon \otimes \eta \otimes \nu$, we find from the transformation rules (3.3) that supersymmetry is

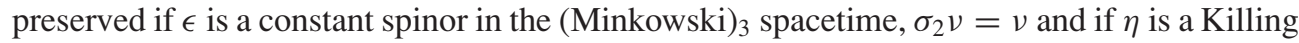
spinor on $S^{3}$, satisfying

$$
\nabla_{i} \eta=\frac{\mathrm{i} g}{\sqrt{2}} \gamma_{i} \eta
$$

Thus the solution has three-dimensional $\mathcal{N}=4$ supersymmetry.

\section{Discussion}

It was shown in [6] that a variant $\mathcal{N}=(1,1)$ supergravity may be constructed on the basis of a generalized Scherk-Schwarz reduction from seven dimensions. In this paper, we have completed the analysis of the fermion sector of this model, and have presented the variant supersymmetry transformations in section 2 . In general, the resulting theory contains two mass parameters, $m_{1}$ and $m_{2}$, and consists of a single vector multiplet coupled to gravity. A further truncation to pure $\mathcal{N}=(1,1)$ supergravity may be obtained by setting $m_{1}=m_{2}$.

It may be seen from $(2.14)$ that the resulting model reduces to ordinary $\mathcal{N}=(1,1)$ ungauged supergravity [9] in the limit $m \rightarrow 0$. In fact, the parameter $m$ is similar to a 
gauging parameter in the sense that a potential $V=25 m^{2} \mathrm{e}^{-\frac{1}{2} \hat{\phi}}$ is generated whenever $m \neq 0$. Nevertheless, this new theory is rather unusual in that the bare vector potential terms in (2.14) do not correspond to the usual minimal coupling to charged fermions. In this sense, the $\mathcal{N}=(1,1)$ theory constructed in [6] differs from conventional models with gauged $R$-symmetry.

In the bosonic sector (with vanishing $\operatorname{Sp}(1)$ singlet gauge field), the field content and equations of motion of the variant $\mathcal{N}=(1,1)$ theory resembles that of the Salam-Sezgin model. In particular, the bosonic Lagrangian (3.1) is identical to that of the gauged $\mathcal{N}=(1,0)$ model, albeit with a triplet of gauge fields. Recall, however, that the $\mathcal{N}=(1,1)$ supergravity multiplet decomposes into a graviton, gravitino and a tensor multiplet of $\mathcal{N}=(1,0)$ supersymmetry. Thus the singlet and triplet gauge fields of the $\mathcal{N}=(1,1)$ supergravity reside in the gravitino multiplet, and not a vector multiplet, as would be necessary for obtaining a Salam-Sezgin truncation. One practical implication of this observation is that, while the present model admits similar vacuum solutions to the Salam-Sezgin model, its supersymmetry properties could in principle be drastically different.

As an example, we have demonstrated that the variant supergravity admits an $M_{4} \times S^{2}$ reduction yielding $D=4, \mathcal{N}=2$ supergravity coupled to a single vector multiplet. Although we have used Weyl notation for the four-dimensional fermions, it is clear that this theory is non-chiral in the usual sense. Thus, while a further truncation to $\mathcal{N}=1$ supergravity coupled to a chiral multiplet is possible, we are unable to obtain a chiral theory in four dimensions through this reduction process.

At this point, it is worth recalling that the presence of Weyl fermions in four dimensions is insufficient to ensure a chiral theory. Since $\mathcal{N}=1$ graviton and vector multiplets are inherently non-chiral, it is necessary to have chiral multiplets transforming under a complex representation of the gauge group in order to obtain a chiral model. Note, in particular, that uncharged fermions may be described in either Weyl or Majorana notation, so the presence of 'left-handed' gravitinos in (3.16) is not an indication of actual chirality.

While the present reduction does not result in massless charged fermions in four dimensions, it should be emphasized that the $M_{4} \times S^{2}$ reduction of the Salam-Sezgin model likewise is non-chiral, although the argument is somewhat subtle. As demonstrated in [10], smooth Kaluza-Klein reductions in the gravitational sector cannot lead to a chiral theory in four dimensions. However, [10] goes on to indicate that chirality may be obtained by starting with chiral fermions coupled to gauge fields in the higher dimensional theory, provided the gauge reduction is non-trivial. In particular, reductions with a monopole flux such as [11] could in principle give rise to four-dimensional chirality. This would suggest that the SalamSezgin model is chiral, since it precisely involves turning on such a $U(1)$ monopole flux, with all fermions charged under this $U(1)$. However, as emphasized in [2, 5], the $U(1)$ does not survive the reduction to four dimensions. The resulting theory contains only $S U(2)$ gauge fields and uncharged fermions, and is hence non-chiral.

Of course, the minimal Salam-Sezgin model in itself is anomalous, and additional $\mathcal{N}=(1,0)$ vector and matter multiplets must be added to cure the anomalies. This presents a natural opportunity to construct a model with six-dimensional charged chiral fermions. Unfortunately, however, to preserve supersymmetry the monopole flux used in the $M_{4} \times S^{2}$ reduction must be that corresponding to the gauging of a $U(1)$ subgroup of $\operatorname{Sp}(1)$. Since it is precisely this $U(1)$ that is absent in four dimensions, the resulting theory will again be nonchiral. This argument does not necessarily preclude the possibility of non-supersymmetric chiral reductions. However, that is not of present interest.

While the $M_{4} \times S^{2}$ reduction in itself is non-chiral, nevertheless chirality may still survive in brane models [3] where chiral families live solely on the branes, and not in the bulk. In 
fact, from a braneworld perspective, the present model provides an alternative framework to the Salam-Sezgin model, where the bulk solution preserves $\mathcal{N}=2$ supersymmetry, and it is the branes themselves that provide both chirality and an additional halving of supersymmetry to $\mathcal{N}=1$. It would be of interest to study the resulting braneworld models constructed from the present theory.

\section{Acknowledgment}

JTL would like to thank the George P \& Cynthia W Mitchell Institute for Fundamental Physics at Texas A\&M University for hospitality during the course of this work. HL and CNP were partially supported by the DOE grant DE-FG02-95ER40899 and JTL by the DOE grant DE-FG03-95ER40917.

\section{References}

[1] Salam A and Sezgin E 1984 Chiral compactification on Minkowski $\times S^{2}$ of $N=2$ Einstein-Maxwell supergravity in six dimensions Phys. Lett. B 14747

[2] Aghababaie Y, Burgess C P, Parameswaran S L and Quevedo F 2003 SUSY breaking and moduli stabilization from fluxes in gauged 6D supergravity J. High Energy Phys. JHEP03(2003)032 (Preprint hep-th/0212091)

[3] Aghababaie Y, Burgess C P, Parameswaran S L and Quevedo F 2003 Towards a naturally small cosmological constant from branes in 6D supergravity Preprint hep-th/0304256

[4] Guven R, Liu J T, Pope C N and Sezgin E 2003 Fine tuning and six-dimensional gauged $N=(1,0)$ supergravity vacua Preprint hep-th/0306201

[5] Gibbons G W and Pope C N 2003 Consistent $S^{2}$ Pauli reduction of six-dimensional chiral gauged EinsteinMaxwell supergravity Preprint hep-th/0307052

[6] Kerimo J and Lü H 2003 New $D=6, \mathcal{N}=(1,1)$ gauged supergravity with supersymmetric Minkowski $\times S^{2}$ vacuum Phys. Lett. B 576219 (Preprint hep-th/0307222)

[7] Townsend P K and van Nieuwenhuizen P 1983 Gauged seven-dimensional supergravity Phys. Lett. B 12541

[8] Salam A and Sezgin E 1983 SO(4) gauging of $N=2$ supergravity in seven dimensions Phys. Lett. B 126295

[9] Romans L J 1986 The $F_{4}$ gauged supergravity in six dimensions Nucl. Phys. B 269691

[10] Witten E Fermion quantum numbers in Kaluza-Klein theory Preprint PRINT-83-1056 (PRINCETON) published in Shelter Island II 1983:227

[11] Randjbar-Daemi S, Salam A and Strathdee J 1983 Instanton induced compactification and fermion chirality Phys. Lett. B 13256 